Presented at and to Appear in Proceedings of the $4^{\text {th }}$ Pedestrian and Evacuation Dynamics Conference, Duisburg Germany, 27-29 Feb 2008.

\title{
Optimising Vessel Layout Using Human Factors Simulation
}

\author{
Steven J. Deere ${ }^{1}$, Edwin R. Galea ${ }^{1,2}$ and Peter J. Lawrence ${ }^{1}$ \\ ${ }^{1}$ Fire Safety Engineering Group, University of Greenwich, London, UK \\ Contact email: ${ }^{2}$ e.r.galea@gre.ac.uk
}

\begin{abstract}
Summary
Evaluating ship layout for human factors (HF) issues using simulation software such as maritimeEXODUS can be a long and complex process. The analysis requires the identification of relevant evaluation scenarios; encompassing evacuation and normal operations; the development of appropriate measures which can be used to gauge the performance of crew and vessel and finally; the interpretation of considerable simulation data. In this paper we present a systematic and transparent methodology for assessing the HF performance of ship design which is both discriminating and diagnostic.
\end{abstract}

\section{Introduction}

When modifying the internal configuration of a ship, it is important to determine what, if any, HF benefits or disbenefits may result. How these aspects can be assessed is less well defined. In this paper we present a novel mathematical procedure, based on computer simulation of evacuation and normal operations (NOP), for assessing the overall HF performance of ship design.

Making modifications to the internal layout of a ship or its operating procedures will have HF implications for crew and passengers, which in turn will have an impact on overall levels of safety under emergency conditions and efficiency of operation in normal conditions. For naval vessels, the location and distribution of compartments may have an impact on the time required by crew to go from one state to another, it may also have an impact on the minimum number of crew required to safely and efficiently operate the vessel under a variety of different conditions. These factors will have an impact on the vessels overall operating efficiency, ability to fulfil the assigned mission and lifetime costs associated with crewing requirements.

Advanced ship evacuation models such as maritimeEXODUS can be used to determine the performance of personnel under emergency conditions for both passenger and naval vessels as well as the normal circulation of personnel for both passenger and naval vessels [1,2]. These models produce a wide variety of simulation outputs, such as time to assemble and the levels of congestion experienced. As the number of different scenarios investigated increases, so does the volume of output data. It therefore becomes increasingly difficult to 
consistently assess changes in HF performance associated with changes in vessel configuration across a wide range of scenarios and performance requirements.

In this paper we explore a methodology to assess changes in HF performance resulting from changes to vessel configuration and/or crew procedures. The approach is intended to be both diagnostic and discriminating. The identified methodology is being developed as part of a collaborative project between the authors and the Design Research Centre (DRC) of University College London, funded by the UK EPSRC with support from MoD.

\section{Methodology for Assessing Human Factors Performance}

In order to gauge the HF performance of the vessel it is essential to define a range of relevant Evaluation Scenarios (ES) against which the vessel will be tested. These scenarios are intended to define the scope of the challenges the vessel will be subjected to. In order to gauge vessel performance across a range of criteria, the ES are made up of both evacuation and NOP scenarios.

Relevant evacuation scenarios may include those required by MSC Circular 1238 [3] and include the IMO night and day scenarios or their naval equivalent [4]. In addition to defining the ES, a range of Performance Measures (PM) must be defined that measure various aspects of personnel performance in undertaking the tasks associated with the ES. PM for passenger ship evacuation scenarios may include the time required to complete the assembly process while for a naval vessel NOP scenario, the total number of water tight doors (WTD) opened and closed may be relevant. The suitability of the vessel layout will be evaluated for fitness of purpose through some combination of the PM resulting from the execution of the ES.

Collectively the particular combination of ES and PM that results in a meaningful measure of the performance of the crew and vessel are described as the Human Performance Metric (HPM). The HPM works by systematically evaluating one layout design against another, whether this is two variants of the same design or two completely different designs. In this paper we will focus on applications involving naval vessels and in particular frigate type surface combatants.

\section{The Components of the Human Performance Metric}

To demonstrate the concept of the HPM we define the key components of the HPM for a naval surface combatant (i.e. a frigate class vessel).

\subsection{Evaluation Scenarios}

NOP scenarios represent situations where the ships crew move around the vessel carrying out specific tasks. An example of a NOP scenario for a naval vessel is the 'State 1 preps'. This scenario disregards the normal non essential tasks and brings the organisation of personnel, equipment, machinery and water tight (WT) integrity to the highest state of preparedness and readiness to deal with any emergency that might occur. 


\subsection{Functional Groups}

As members of the ships complement may be involved in undertaking different tasks during a particular ES, the ships complement is divided into subgroups. Membership of each subgroup is determined by the nature of the tasks undertaken by the individuals in the particular ES, with each subgroup being made up of people undertaking a common set of tasks. These subgroups are labelled Functional Groups (FG). An example of a FG is the 'damage control and fire fighting' (DCFF) group which is a prime example of a FG used in circulation ES. In addition to the FGs defined by specific sub-populations, a special FG, identified as Ships Company, is included in all ES. This paper will make use of the FG; 'Entire Ships Company' and 'Damage control and fire fighting'.

\subsection{Performance Measures}

To assess the performance of each FG in each ES, a set of Performance Measures (PM) have been defined, each of which uniquely assesses a particular aspect of the scenario. Each of the PMs returns a value determined from the computer simulation of the ES which is then used in part to complete the HPM. The higher the value of the PM, the poorer the performance of the FG in the ES. Some 31 PM have been defined which assess many aspects of crew performance for a frigate.

\section{Defining the Human Performance Metric}

The HPM is used to compare the human performance capabilities of competing vessel designs. These alternative designs may simply be different design iterations of a particular vessel or competing design options. To assess the performance of the vessels, a set of evaluation scenarios are selected which are relevant to the intended operation of the vessel. The design alternatives are then crewed with the required number of personnel and the crew assigned to their functional groups. The number and type of FG may differ between design alternatives for each ES. Finally, each functional group has a set of performance measures defining the performance of the FG.

Each PM extracts its value from maritimeEXODUS simulation software. The $\mathrm{PM}$ are then normalised in order to carry out a direct comparison between designs. The weighted sum of the normalised PM values produces the FG score. The weighted sum of the FG scores produce the ES score and the weighted sum of the ES scores produce the overall vessel performance (VP) score. The overall Vessel Performance (VP) for design X can then be compared against the VP score for all other designs to determine which design produced the best overall performance. The matrix is also diagnostic in that it allows the identification of which measures contributed to the poor performance of a failed vessel design, or which PM could be improved in a winning design.

\section{Demonstration Application of the HPM}

The use of the HPM concept in evaluating the relative performance of two designs of a hypothetical naval vessel will be demonstrated in this section. To do this, seven ESs are considered, three evacuation scenarios and four NOP. 
The aim of this analysis is to determine which design variant is the most efficient in terms of its HF performance and whether any improvements to the winning design can be identified.

\subsection{The Geometry}

The baseline vessel design (variant 1) consists of 453 compartments spread over eight decks. Decks No 1 and No 2 (deck 4 and 5 respectively) have a single central passageway connecting the aft to forward section of the deck. The second variant design (variant 2) consists of the 445 compartments spread over eight decks as in variant 1 . The key difference between the two designs is that variant 2 has two passageways running in parallel from the aft to the forward end of the vessel on both decks.

\subsection{The Scenarios}

Each vessel has a complement of 262. The crew are initially located in the location they would be expected to be at the start of each scenario as determined by the "state" of the vessel. Crew members not on watch are located in their cabin. The seven ES used to assess the performance of each vessel are; 'normal day cruising A', 'normal day cruising B', 'Action Station evacuation', 'State 1 Preps', 'Blanket Search', 'Family Day A' and 'Family Day B' scenarios. It must be noted that the scenarios used in this demonstration are not intended to accurately represent actual naval operations, but are used simply to demonstrate the HPM concept.

\subsection{The simulation software}

The ship evacuation model maritimeEXODUS [1,2] produced by FSEG was used to perform the personnel simulations presented in this paper. The software has a number of unique features such as the capability to represent the performance of both naval personnel and civilians in the operation of watertight doors, vertical ladders, hatches and 60 degree stairs. Another feature of the software is the ability to assign passengers and crew a list of tasks to perform. In addition, a separate utility program has been developed (the Human Performance Metric Analyser) which automatically constructs the matrix of human performance scores from maritimeEXODUS output that are used in the evaluation of the vessel design.

\subsection{Results and Analysis}

The seven ES were each run 50 times and representative simulation result files were selected for each scenario to construct the HPM for each variant. The PMs for each variant were then determined and the final HPM constructed for each variant as shown in Table 1.

As can be seen from Table 1, Variant 1 produces an overall Vessel Performance (VP) score of 523.7 while Variant 2 produces a VP score of 531.2. Thus we note that the overall performance of both variants is broadly similar, with Variant 1 producing a marginally better $(1.4 \%)$ overall human factors performance according to the measures we have identified. Furthermore, we note that Variant 2 outperformed Variant 1 in most of the scenarios, however Variant 1 significantly outperformed Variant 2 in two 
Presented at and to Appear in Proceedings of the $4^{\text {th }}$ Pedestrian and Evacuation Dynamics Conference, Duisburg Germany, 27-29 Feb 2008.

NOPs and the worst performing scenario for Variant 1 is the 'Action Stations Evacuation'. As Variant 1 produces the better overall performance and produces significantly better NOPs performance it would be consider the design of choice. However, its performance may be improved by investigating why it did poorly in its worst performing ES.

Table 1. Scenario Scores for Variant 1 and Variant 2

\begin{tabular}{||l|c|c|c|c||}
\hline \multicolumn{1}{|c|}{ Evaluative scenario } & $\begin{array}{c}\text { Scenario } \\
\text { Weight }\end{array}$ & $\begin{array}{c}\text { Variant } \\
1\end{array}$ & $\begin{array}{c}\text { Variant } \\
2\end{array}$ & $\begin{array}{c}\text { \% difference between } \\
\text { Variant 1 and Variant 2 }\end{array}$ \\
\hline \hline Normal Day Cruising A & 1 & 46.14 & 44.33 & $3.93 \%$ \\
\hline Normal Day Cruising B & 1 & 50.81 & 46.79 & $7.92 \%$ \\
\hline Action Stations Evacuation & 1 & 51.45 & 46.70 & $9.23 \%$ \\
\hline State 1 Preps & 1.5 & 67.46 & 75.47 & $-11.87 \%$ \\
\hline Blanket Search & 1.5 & 78.04 & 84.29 & $-8.01 \%$ \\
\hline Family Day A & 1.5 & 48.65 & 47.20 & $2.99 \%$ \\
\hline Family Day B & 1.5 & 56.03 & 55.32 & $1.26 \%$ \\
\hline \hline \multicolumn{2}{|r|}{ Overall Performance of design } & 523.7 & 531.2 & \multicolumn{1}{|c|}{} \\
\hline
\end{tabular}

It must be emphasised that this conclusion is based on the particular Evaluation Scenarios, Performance Measures and Weights that have been used in the analysis. To better understand why Variant 2 out performed Variant 1 in the 'Action Stations Evacuation' scenario and to identify potential areas in which Variant 1 can be further improved it is necessary to delve into the sub-components of the HPM. Presented in Table 2 are the PM scores for Variant 1 and 2 for this scenario.

Table 2. Variant 1 and Variant $2 P M$ results for $\mathrm{FG}_{1}$ in $\mathrm{ES}_{3}$.

\begin{tabular}{|l||c||c|c||c|c||}
\hline \multicolumn{2}{|c||}{ FG $_{1}$ - Entire Ships Company } & \multicolumn{2}{c|}{ Variant 1 } & \multicolumn{2}{c||}{ Variant 2 } \\
\hline $\begin{array}{l}\text { C1 - number of locations in which pop density } \\
\text { exceeds 4 p/m }{ }^{2} \text { for more than 10\% of overall } \\
\text { scenario time' }\end{array}$ & 8 & 4 & 1 & 4 & 1 \\
\hline $\begin{array}{l}\text { C2 - the max time the pop density exceeded the } \\
\text { regulatory max 4 p/m² for 10\% of sim time }\end{array}$ & 3 & 75.40 & 1 & 42.14 & 0.56 \\
\hline $\begin{array}{l}\text { G1 - average time required to complete } \\
\text { operations }\end{array}$ & 4 & 256.7 & 1 & 193.54 & 0.75 \\
\hline G2 - average time spent in transition & 3 & 36.61 & 0.80 & 45.76 & 1 \\
\hline G3 - time to reach final state & 8 & 666.7 & 0.22 & 594.50 & 0.20 \\
\hline G4 - Average time spent in congestion & 3 & 150.6 & 1 & 74.93 & 0.50 \\
\hline G5 - average distance travelled & 4 & 47.11 & 0.94 & 50.11 & 1 \\
\hline M1 - the number of WTD used during scenario. & 2 & 24 & 0.89 & 27 & 1 \\
\hline $\begin{array}{l}\text { M8 - the number of times the FG moved } \\
\text { between decks }\end{array}$ & 2 & 373 & 1 & 322 & 0.86 \\
\hline M16 - Average number of doors used/person & 3 & 1.59 & 0.82 & 1.94 & 1 \\
\hline M17 - Average number of WT doors/person & 3 & 1.46 & 1 & 1.19 & 0.82 \\
\hline M18 - Average number of hatches used & 3 & 0.27 & 1 & 0.23 & 0.83 \\
\hline
\end{tabular}

We note that for Variant 2, the overall average time spent in congestion (as measured by $\mathrm{G}_{4}$ ) was some $50 \%$ less than in Variant 1 . This significant 
reduction in congestion results in Variant 2 being able to complete the scenario $11 \%$ quicker than Variant 1 (as measured by $\mathrm{G}_{3}$ ). Indeed, we note that while both vessels easily satisfy the international set evacuation time requirements (as measured by $\mathrm{G}_{3}$ ) the levels of congestion experienced exceed the international set limits in four locations (as measured by $\mathrm{C}_{1}$ ) and Variant 1 experiences the most severe congestion (as measured by $\mathrm{C}_{2}$ ). To address this issue and to improve the overall performance of Variant 1, further investigation is required to uncover the causes of the severe congestion. Exploring the areas of congestion in Variant 1 suggested that a single additional ladder connecting 01 Deck with No 1 Deck between two of the severe congestion regions may alleviate some of the congestion by providing an additional means of vertical movement. With this modification in place the HPM was re-evaluated for the Modified Variant 1. The Modified Variant 1 now outperforms the original Variant 1 in each scenario and produces an overall VP which is 6\% more efficient than the original Variant 1 and 8\% more efficient than the Variant 2 design. We also find that the Modified Variant 1 design outperforms the Variant 2 design in all but the 'Normal Day Cruising A' evacuation scenario.

\section{Concluding Comments}

This paper has described and demonstrated a general methodology, the Human Performance Metric (HPM), for evaluating HF performance of competing ship designs. The approach is both systematic and transparent allowing user priorities to be clearly stated as part of the methodology. The user priorities can be identified through the selection of appropriate evaluation scenarios and the weights assigned to the various components of the HPM. The methodology is intended to be used as a comparative tool, where the performance of one variant is compared with the performance of an alternative variant.

\section{Acknowledgements:}

The authors gratefully acknowledge the support of the UK EPSRC under grant GR/T22100/01 and the Directorate of Sea Systems, Defence Equipment and Support organisation of the UK MoD (DES-SESea) and our partner organisation DRC of UCL.

\section{References:}

1. S.J.Deere, E.R.Galea, P.J.Lawrence, and S.Gwynne. The impact of passenger response time distribution on ship evacuation performance. The Transactions of The Royal Institution of Naval Architects, Vol. 148, Part A1 (Journal of Maritime Engineering), 2006, pp 35-44.

2. P. Boxall, S.Gwynne, L.Filippidis, E.R.Galea, and D.Cooney, Advanced Evacuation Simulation Software and its use in Warships. Proc of the Human Factors in Ship Design, Safety and Operation. London UK, 23-24 Feb 2005, Publishers: The Royal Institute of Naval Architects, pp 49-56

3. International Maritime Organisation. Guidelines for Evacuation Analysis for New and Existing Passenger Ships, IMO MSC.1/Circ. 1238, 2007.

4. NATO Naval Armaments Group, Maritime Capability Group 6. Chapter VII Escape Evacuation and Rescure, ALLIED NAVAL ENGINEERING PUBLICATION 'ANEP - 77', NAVAL SHIP CODE, 2006. 